\title{
Metrology for trending alignment of the James Webb Space Telescope before and after ambient environmental testing
}

Theo Hadjimichael ${ }^{1}$, Raymond G. Ohl ${ }^{1}$, Josh Berrier ${ }^{6}$, Jeff Gum ${ }^{1}$, Joseph Hayden ${ }^{4}$, Manal Khreshi ${ }^{1}$, Kyle McLean ${ }^{1}$, Kevin Redman ${ }^{5}$, Joseph Sullivan ${ }^{3}$, Greg Wenzel ${ }^{5}$, William Eichhorn ${ }^{2}$, Jerrod Young ${ }^{1}$

${ }^{1}$ NASA Goddard Space Flight Center, ${ }^{2}$ Genesis Engineering Solutions Inc, ${ }^{3}$ Ball Aerospace,,${ }^{4}$ Sigma Space Corporation, ${ }^{5}$ Sierra Lobo, Inc, ${ }^{6}$ Tech Solutions

\begin{abstract}
NASA's James Webb Space Telescope (JWST) is a $6.6 \mathrm{~m}$ diameter, segmented, deployable telescope for cryogenic IR space astronomy. The JWST Observatory architecture includes the Optical Telescope Element (OTE) and the Integrated Science Instrument Module (ISIM) element which contains four science instruments (SIs). Prior to integration with the spacecraft, the JWST optical assembly is put through rigorous launch condition environmental testing. This work reports on the metrology operations conducted to measure changes in subassembly alignment, including the primary mirror segments, the secondary mirror to its support structure, the tertiary mirror assembly to the backplane of the telescope and ISIM.
\end{abstract}

Keywords: JWST, Metrology, Optical alignment, Laser Radar

\subsection{INTRODUCTION}

The James Webb Space Telescope (JWST) is a NASA mission with diverse objectives in astrophysics developed in partnership with the European and Canadian Space Agencies ${ }^{1}$. The JWST’s optical train consists of a cryogenic $(\sim 40 \mathrm{~K})$ Optical Telescope Element (OTE) with a 6.6m diameter primary mirror and a suite of four, cryogenic near- and midinfrared (IR) instruments. The science instruments (SIs) are: Near-Infrared Camera (NIRCam), Near-Infrared Spectrometer (NIRSpec), Mid-Infrared Instrument (MIRI), and Near-InfraRed Imager and Slit-less Spectrograph (NIRISS). The instrument suite also includes the Fine Guidance Sensor (FGS Guider). Together, the SIs and supporting subsystems are the Integrated Science Instrument Module (ISIM) element which is integrated to the back of the OTE. Other components of the JWST observatory include a spacecraft and a sunshade that provides part of the passive cooling system for the science payload.

In the fall of 2016 the ISIM was integrated to the OTE and met its ambient alignment requirements. ISIM had undergone rigorous characterizations and environmental testing on its own ${ }^{1,2,3,4,5,6}$. After ISIM and the OTE (OTIS) were integrated, the OTIS was required to experience both vibration (for three orientations) and acoustics testing. Pre- and post-environmental metrology was performed to determine whether any shifts had occurred between key subsystems due to the exposure. These measurements were conducted before and after the entirety of the environmental testing with a contingency plan for possible tests between exposures if determined necessary.

This paper describes metrology performed on targets/references and surfaces on the OTIS. This includes the Backplane Support Fixture Master References (BSF MR), the Aft Optics Subsystem (AOS), the ISIM targets and other support fixture metrology references and "tie-point" targets. Laser radar (LR) was our primary measurement tool. The LRs measured stationary metrology targets and is capable of direct surface "scanning." We also used Laser trackers (LT) to augment the LR and cross check the LR's target measurements. Like the LR, the LT measures stationary targets and also has the ability to "track" dynamic or moving metrology targets ${ }^{7,8}$. 
The metrology targets are specific to the type of instrument used --- we used high quality, steel tooling balls (TB) and spherically mounted retro-reflectors (SMR) for LR and LT metrology, respectively. A TB is a low-cost, high-quality, specular, steel sphere with a high-accuracy diameter and is the standard metrology target used for the LR.

The LT requires an optical retro-reflector target, which is provided by the SMR. It is similar to a TB --- a specular steel sphere --- but has an embedded retro-reflector optic or "corner cube" mirror arrangement with its optical vertex coincident with the center of the sphere to high accuracy. Notably, the outer spherical SMR surface can also be measured directly by the LR using the LR's TB algorithm, allowing both instruments to measure the same SMR target, but from different angles or sides, arriving at nominally the same result for the target position.

TBs and SMRs typically attach magnetically to metrology "nests," which are fitted via pins to high-precision tooling holes on the OTIS/ISIM structures. Nests have a prescribed precision offset and targets sit on three points in the nest. Nest are highly repeatable for both pin placement and target placement, typically $\sim 5-10$ microns. In locations with critical drop hazard, particularly on the OTIS, SMRs were used exclusively with safety lanyards connecting the target and nests. The OTIS nests used a combination of magnetic attachment and nests with threads instead of pins (depending on location) to attach them to the hard point target locations on the structure. The ISIM locations utilized integral mounting shank tooling balls with magnetic bases, which securely attached them to the Invar target-nest mounting positions with precision tooling holes closely fitted to the target mounting shank diameter.

\subsection{METROLOGY TARGET AND SUBSYSTEM DESCRIPTIONS}

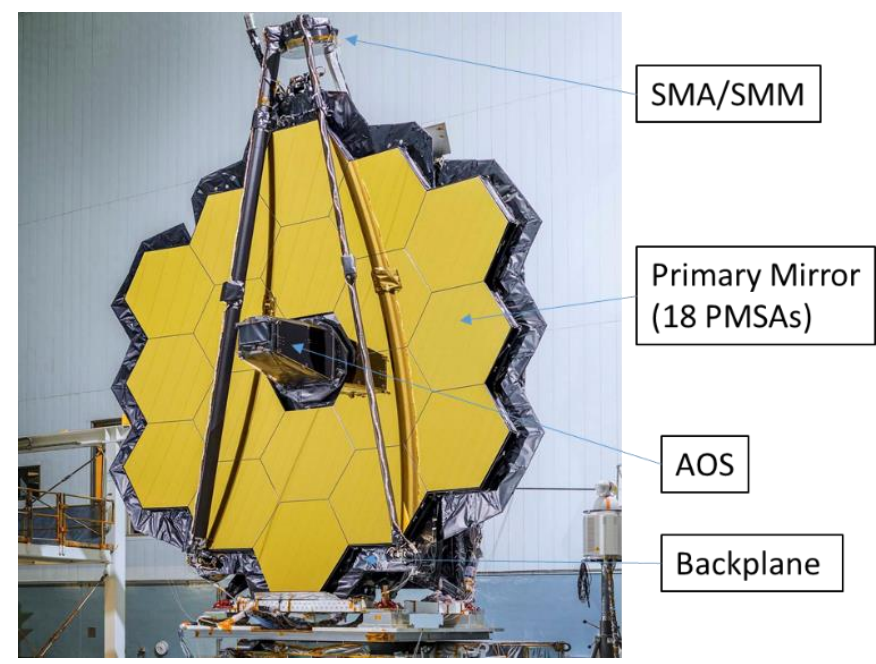

Figure 2-1 The OTIS and subsystems for pre/post-environmental metrology. The ISIM and master reference are mounted behind the primary mirror segments. Photo credit [C. Gunn, GSFC]

\subsubsection{The AOS and Targets}

The AOS holds the OTE's fine steering mirror (FSM) and tertiary mirror. Installed in the center of the telescope backplane, it directs light from the secondary mirror into the instruments on the ISIM. The AOS also defines the telescope coordinate system. Located on the front bulkhead of the AOS are four, 12.7mm diameter SMR targets (with lanyards for drop safety). On the base of AOS are six, $12.7 \mathrm{~mm}$ diameter SMR targets (with lanyards). These SMR targets are pointed towards the LTs and away from the LRs during testing. 


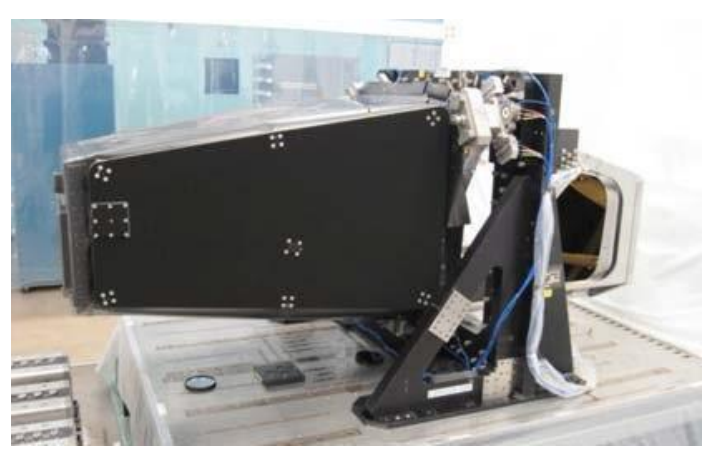

Figure 2-2 Aft Optical System (AOS)

\subsubsection{ISIM Targets}

The ISIM, integrated to the OTE behind the primary mirror, houses the science instruments. During the OTIS metrology testing, only a subset of the original targets used during ISIM characterization and testing were available. Many were unavailable, because, after the ISIM-to-OTE integration, access and lines of sight were reduced. In some areas, thermal blanketing was temporarily removed for this metrology to allow better access to some targets prior to final blanket closeout.
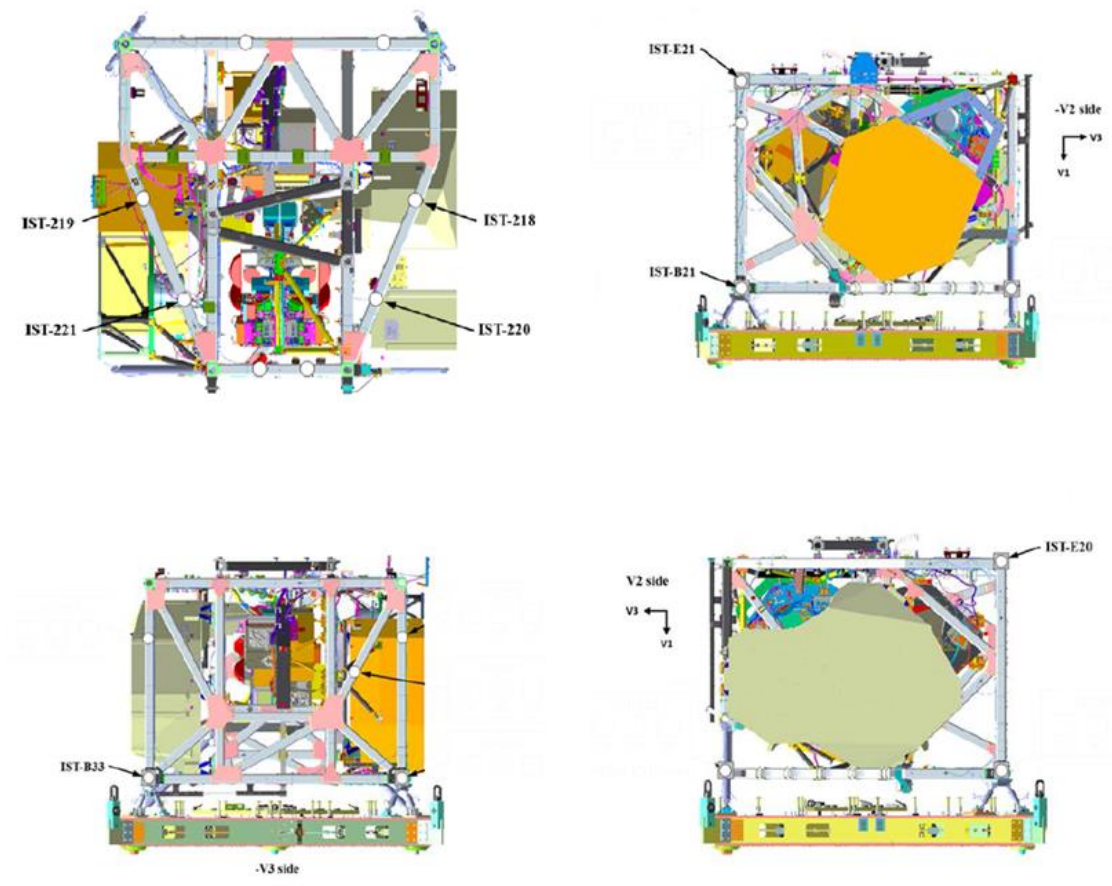

Figure 2-3. ISIM Prime ITOR target locations

Eight integral, monolithic, $12.7 \mathrm{~mm}$ diameter TB targets with magnetic bases were used on the ISIM. These were the only remaining, accessible ISIM target locations during this metrology evolution. These targets were held in place during metrology by their magnetic bases attached to the Invar target mounts on the ISIM structure. The target center mounting offset of the integral TB targets matches the earlier ISIM development metrology TB targets, which utilized separate, $38.1 \mathrm{~mm}$ diameter TBs and bolt-on nests (with lanyards). 


\subsubsection{OTIS Backplane Targets}

The backplane targets, Inboard Hinge (IH) and Dual Hinge (DH), are located at the top and bottom of the primary mirror sections on the primary mirror backplane support structure (PMBSS). They are $12.7 \mathrm{~mm}$ diameter SMR targets (with lanyards) and located near the Secondary Mirror Support Structure (SMSS) leg attachment/hinge points on the PMBSS. These targets use nests attached magnetically to Invar "washers" on the PMBSS. The SMR targets also attached magnetically to the nests.

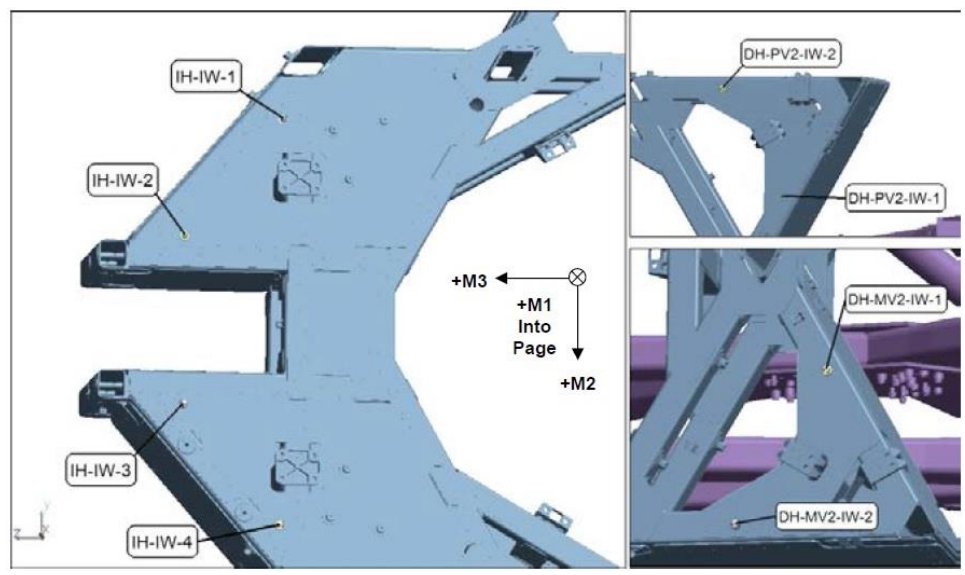

Figure 2-4 PMBSS cross check targets

\subsubsection{Secondary Mirror Assembly Targets}

Five secondary mirror target locations and four were used during this metrology. These are all $12.7 \mathrm{~mm}$ SMR targets with lanyards and separate nests. The targets attached magnetically to the nests.

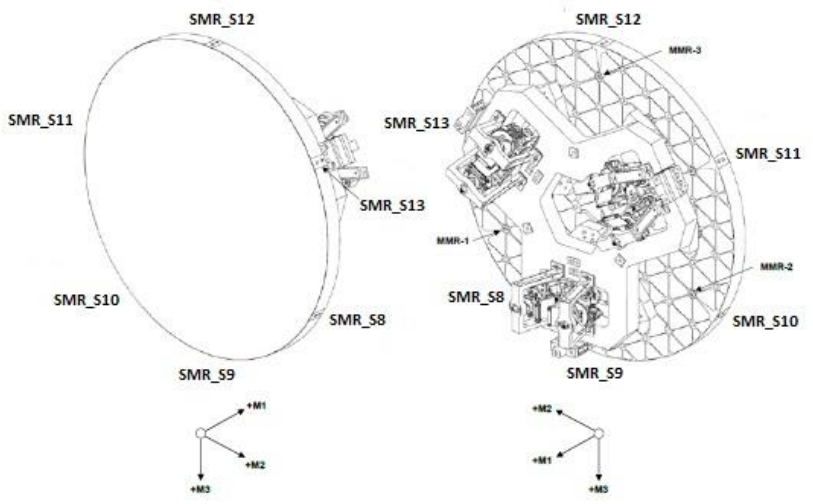

Figure 2-5. SMA target locations 


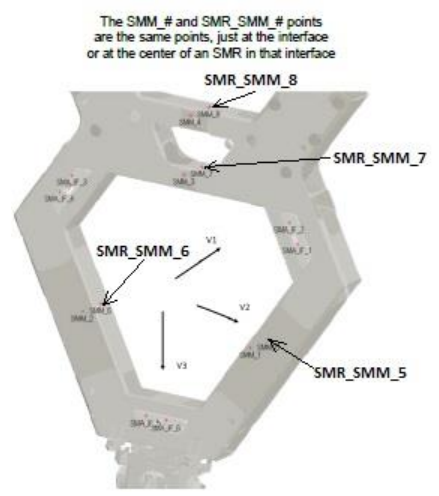

Figure 2-6. SMM target locations

\subsection{METROLOGY OPERATIONS}

There are four different comparisons of alignment change between subsystems. These are 1) the ISIM-to-BSF master reference 2) the AOS-to-Backplane 3) the SMA-to-SMM and 4) the delta PMSA Gap metrology. This last metrology is a direct scan measurement of the gap between two mirrors using a LR to make sure that there are no changes to each mirror position with respect to its neighbor that may adversely affect the mirror ability to deploy and survive the rigors of launch vibration. Each of these subsystems will be measured both during pre- and post-environmental metrology operations. Each group was assigned a local coordinate system parallel to the telescope coordinate system, but with an origin centered on one of the two subsystems being measured. The ISIM to BSF master reference and AOS to backplane was measured during one operation. Line of sign limitations moved us to perform the SMA to SMM measurement in a second configuration with the telescope rotated with optical axis down. The PMSA gap measurements were measured in yet another configuration with the OTIS wings stowed.

\subsection{METROLOGY SETUP, PRE AND POST ENVIRONMENTAL TESTING}

\subsubsection{ISIM and AOS Metrology}

The pre/post-environmental ISIM metrology setup featured the OTIS mounted onto a handling and integration fixture (HIF) attached at the spacecraft interfaces and supported on a roll-over table. The primary mirror "wings" were in the deployed state and the SMA was in the stowed position. During this metrology, instrument stations were established to fully sweep 360 degrees around the OTIS to capture and bundle all targets. LRs and LTs were mounted to metrology stands in a height range of $3 \mathrm{~m}$ to approximately $9 \mathrm{~m}$ in height. 


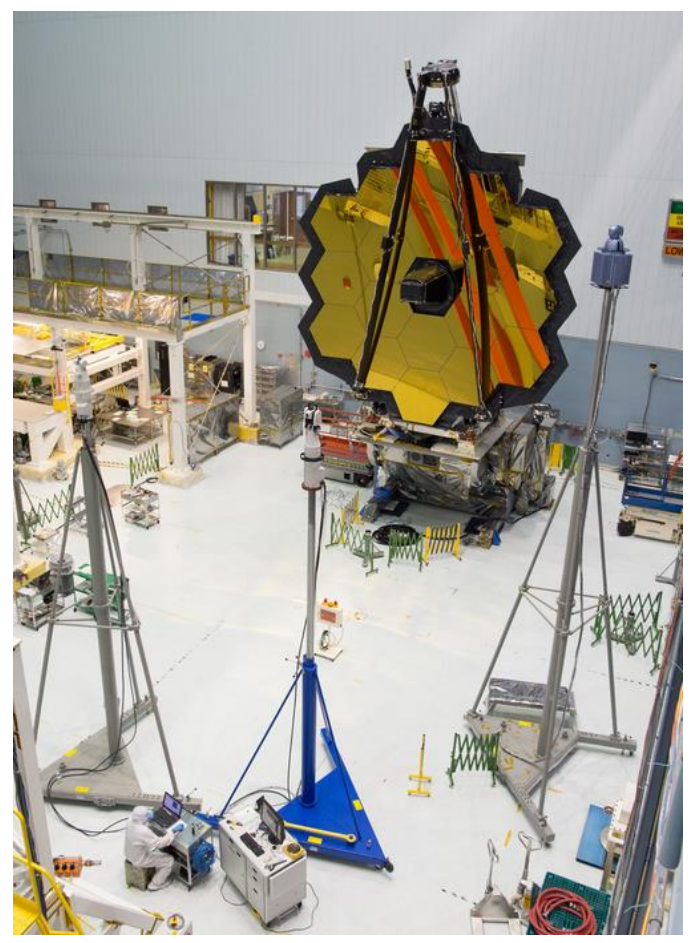

Figure 4-1 OTIS pre/post-environmental metrology setup on rotary table showing some LR and LT stations, Photo credit [C. Gunn, GSFC]
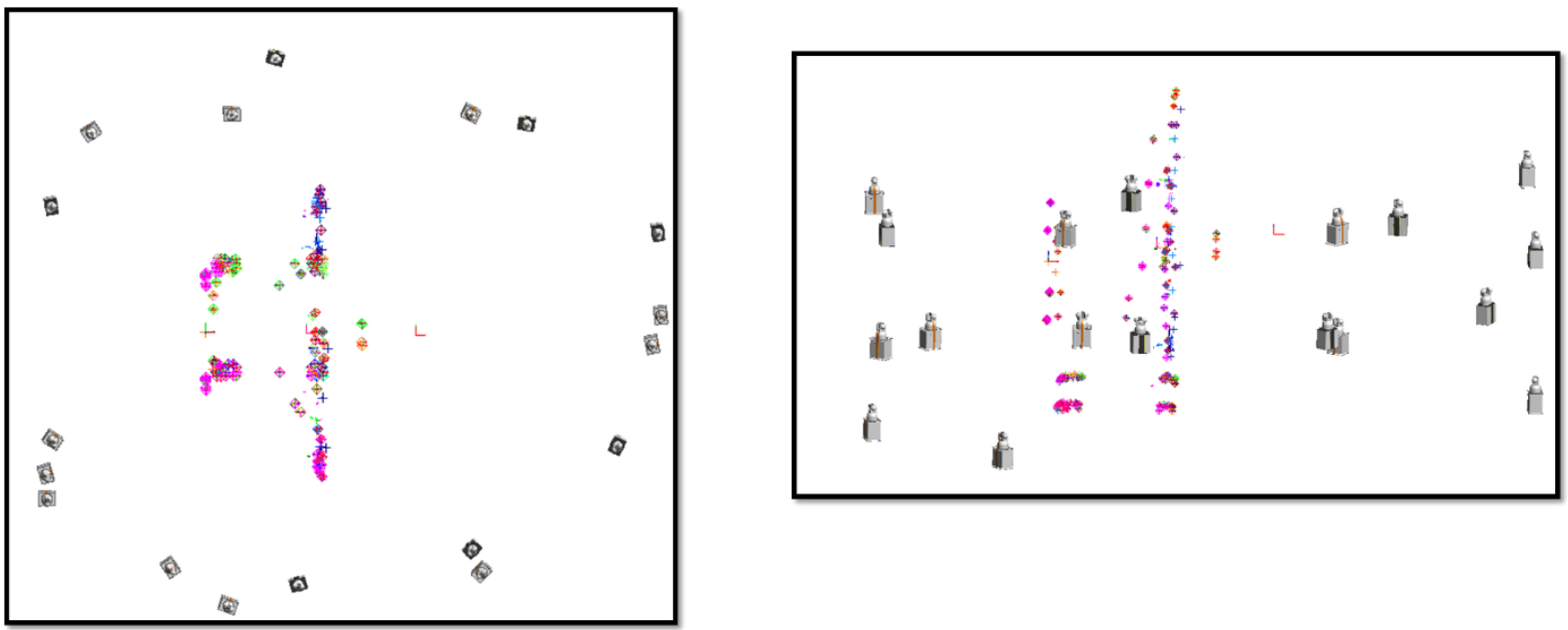

Figure 4-2 typical LR/LT survey of targets top and side views respectively

\subsubsection{SMA-SMM Metrology}

For this metrology, the OTIS was mounted onto the HIF/rotary table and rotated 90 degrees such that the primary mirror surface was approximately parallel with the SSDIF floor (i.e., optical axis down). This provided access to install the SMA-SMM targets and suitable lines of sight for multi-station metrology. For the SMA-SMM metrology, the PMSA wings remained in the "deployed" configuration. Approximately 10 stations were needed to fully characterize the SMA to SMM configuration pre- and post-environmental testing. 


\subsubsection{PMSA Stowed Gap Metrology}

The PMSA gap is defined as the distance between two adjacent PMSA mirror aperture "vertices," where a vertex is the tip of each hexagon-shaped aperture. For this metrology evolution, the OTIS was mounted onto another fixture, the vibe fixture (VF), and supported on a large dolly leveled at three points with high capacity jacks on the floor (Figure 4-2). The dolly was used to transport the OTIS from the cleanroom (in a custom made tent also attached to the dolly though not in place during this metrology) to the various environmental testing areas. During the PMSA gap metrology, the PMSA wings and the SMA were in the "stowed" configuration as shown in Figure 2-13. Approximately 20 stations were needed to fully wrap around the entire OTIS bundling targets front to back. Metrology was performed on the BSF and AOS targets. Additional targets were placed around the base of the telescope on the dolly and VF as well as some PMSA wing targets which provided height to assist an acceptable LR station bundle. The PMSAs themselves had no targets on their substrates or elsewhere. LR vision scans were analyzed at each PMSA vertex (figure 4.4). Since the gaps between each mirror were on the order of millimeters, the approximate LR station locations were based on a line of sight study using a simulation using metrology software (Spatial Analyzer and Measurement Plan; MP) ${ }^{9}$. Each scan needed to acquire three planes (two on the sides of the mirror and the mirror's optical surface) to define a mirror vertex point.

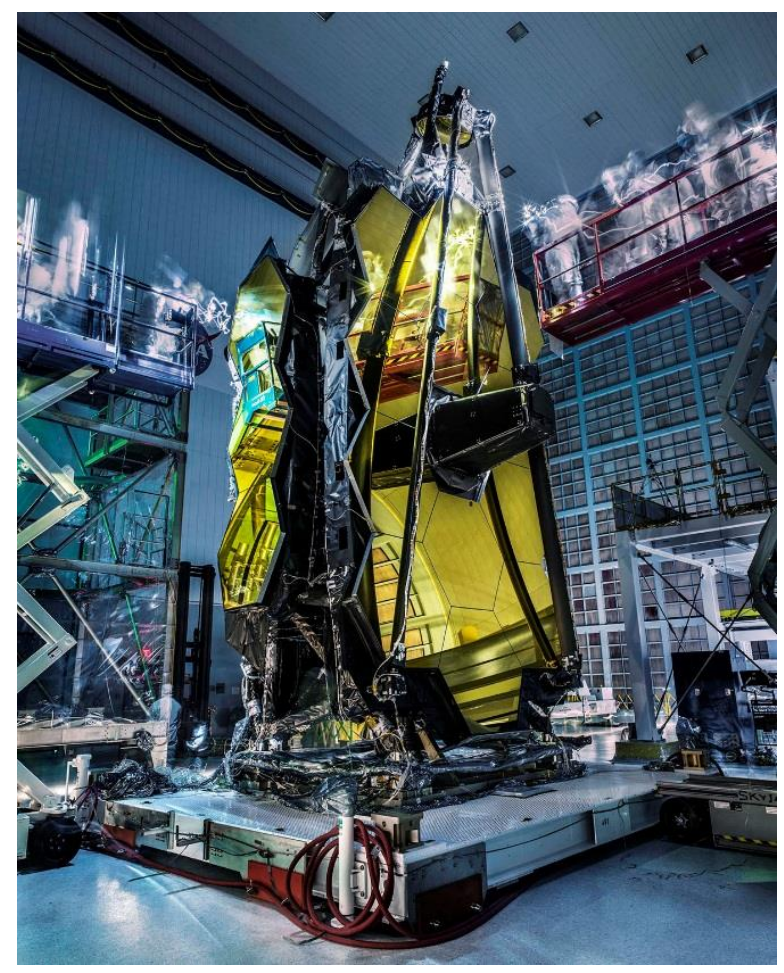

Figure 4-3 PMSA stowed gap metrology configuration on dolly. Photo credit [C. Gunn, GSFC] 


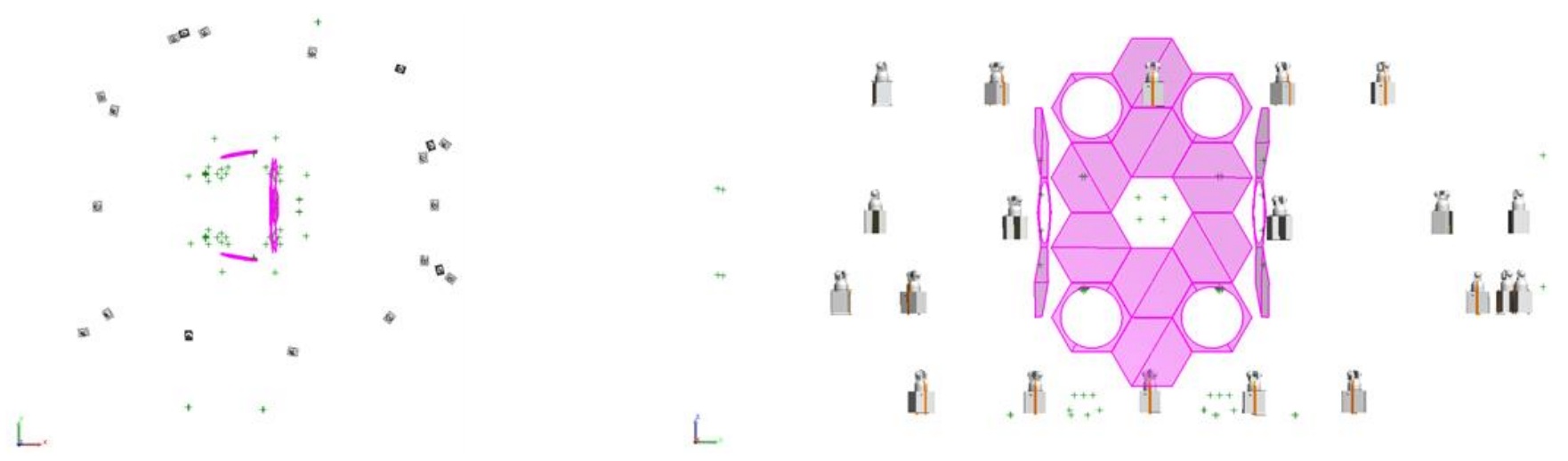

Figure 4-4 Example LR station suite for Gap metrology. a. View from above. b. View from the front of the primary mirror.
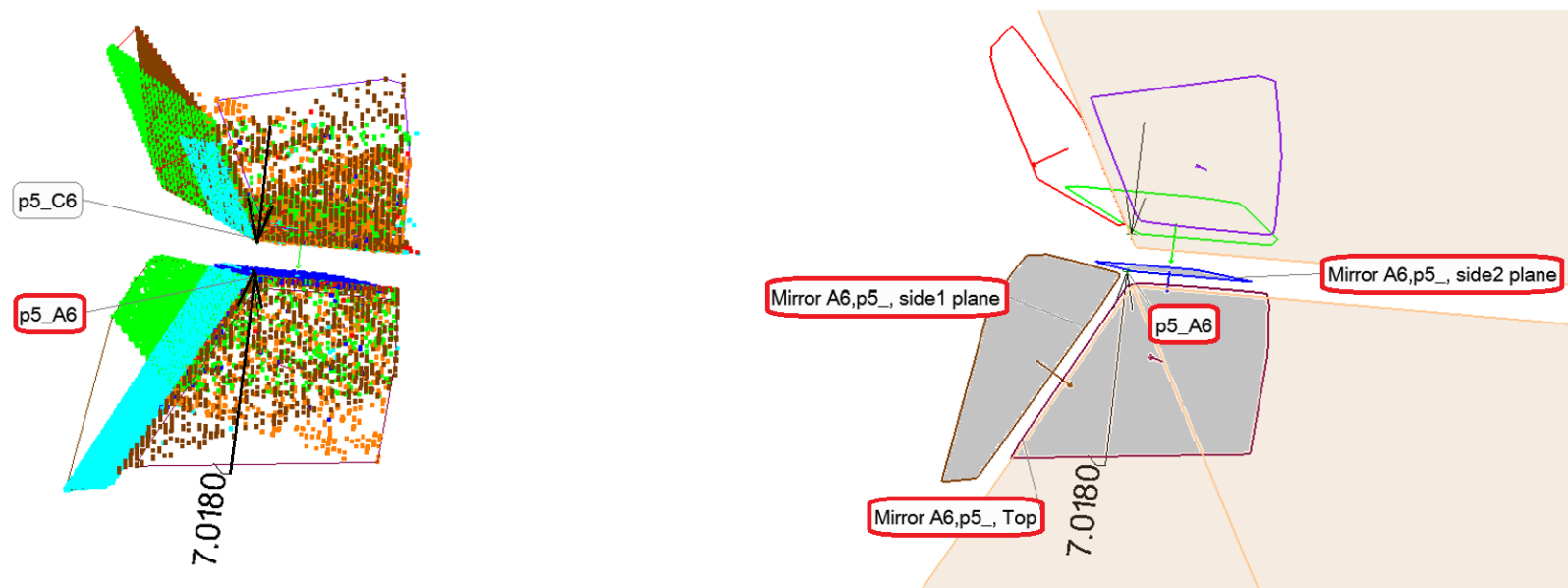

Figure 4-5 example scan output and associated fit planes to determine a mirror vertex

\subsection{METROLOGY DATA ANALYSIS}

\subsubsection{OTIS Subsystem metrology comparisons}

All metrology operations for the pre/post environmental testing followed the same basic procedure. The first step was to acquire all targets (mentioned in previous sections) to a degree such that the uncertainties on each point was at an acceptable minimum. This was especially difficult for the ISIM targets which were mostly buried in the back of the telescope behind thermal blanketing. Even though accommodations were made for lines of sight by strategically folding back corners etc. the target accessibility didn't always meet our goal of capturing every target from at least 3 stations, each station at least 30 degrees away from the others. In the end the overall suite of targets in each subsystem did pass its six degree of freedom transformation uncertainty predictions on the pre/post change. Each LR/LT station captured all targets in its line of sight and repeated the measurement a total of 5 times. As noted above enough stations to completely circumnavigate the telescope was required to fully characterize the telescope before and after environmental testing.

After all targets had been acquired from all LR/LT stations the data from each station was bundled together in Spatial Analyzer TM (SA). Within SA is an operation called the unified spatial metrology network (USMN) ${ }^{10,11}$ which bundles (as individual rigid bodies) each LR/LT station data and their associated uncertainties to form a composite dataset with uncertainty. Our analysis approach uses this composite output for each metrology operation as the baseline dataset. The 
associated uncertainties are developed from an RSS of the five dataset USMN uncertainty, the individual USMN uncertainties (one for each of 5 datasets) and that associated with removing and replacing TB/SMR targets.

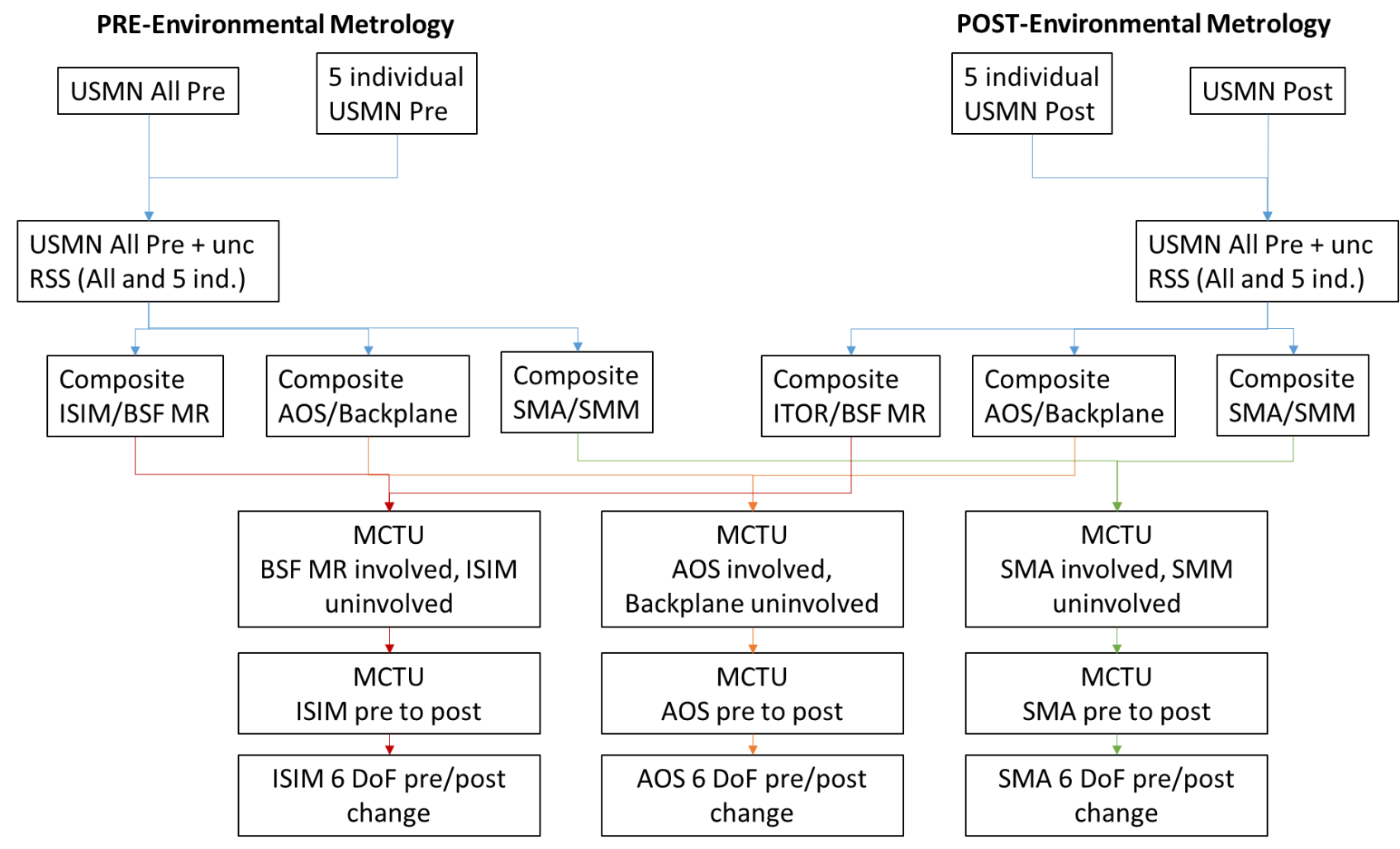

Figure 5-1 ISIM-BSF, AOS-Backplane, and SMA-SMM analysis flow chart

Next, breaking the data down into the subsystem groups mentioned above, a local coordinate system is built at the center of the BSF, AOS, and SMM respectively for the pre-environmental test data. Each subsystem group of two must then be transformed onto each other, into the same coordinate system. For example the pre-environmental BSF targets get a local coordinate system parallel to the telescope coordinate system with origin at the center of mass of the BSF composite targets from the USMN. The post-environmental BSF/ISIM targets are transformed using Monte Carlo Transformation Uncertainty (MCTU) code ${ }^{12}$ to the pre-environmental data using the BSF targets only. Once the two datasets are in the same local coordinate system another Monte Carlo transformation between the pre and post ISIM data determines the six degree of freedom change (if any) of the ISIM with respect to the BSF pre to post environmental testing. Similarly this dataflow (fig 5-1) is used to determine the six degree of freedom pre/post changes for each subsystem of interest.

\subsubsection{Mirror Gap metrology}

As stated before all stations from this metrology operation are bundled together with uncertainties using the USMN function in SA. This bundling not only moves the targets as rigid bodies but also the LR scans associated with each station. The final product brings all the scans together from all the different LR stations such that all mirror scans from different angles of incidence fall on top of each other and 3 surfaces for each mirror vertex on all mirrors can be built. Once all scans are in the same coordinate system using USMN a best fit plane is constructed on each surface of interest. The intersection of three surfaces form a vertex (fig 4-3), one of six on each PMSA. The delta between adjacent vertices 
on an adjacent PMSA closest vertex is defined as the gap. The change in this delta pre to post environmental testing is the reported result. Approximately 150(190) vertex point clouds were collected in pre(post) metrologies. Vertex measurement repeatability was determined during the pre-environmental PMSA Gap metrology trial where several PMSA vertices were measured by repeating scan data acquisition from 2-3 LR stations per approximate location, and analyzing combinations of inter-station data. Each PMSA set of six vertices were also transformed to each other individually to determine any out of family vertex points. Since the PMSA itself is highly unlikely to have distorted this transformation will show any anomaly in the acquisition or analysis of data. In the results below these are pointed out by dashed boxes (see section 6.1.5)

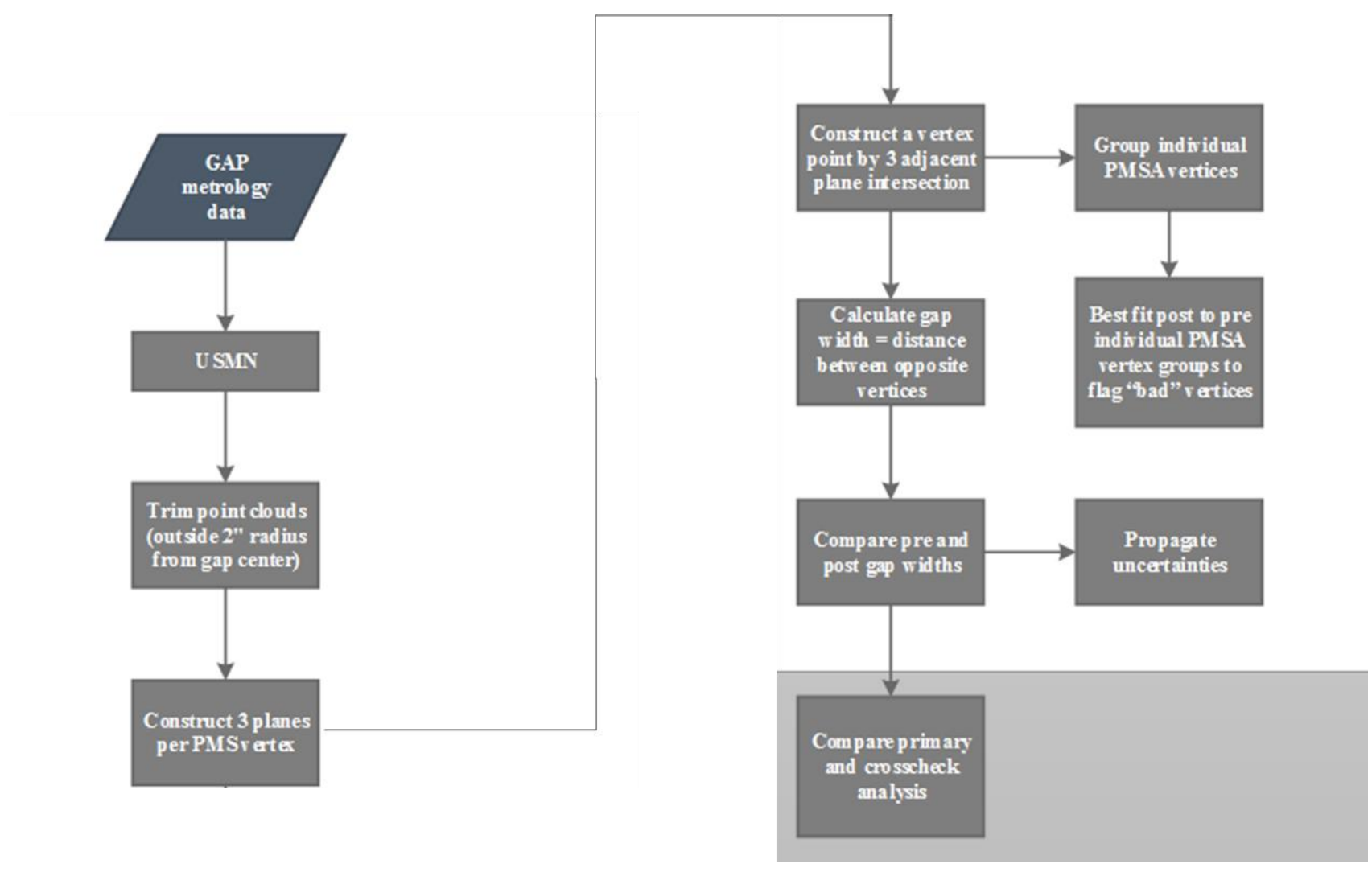

Figure 5-2 Gap metrology analysis flow

\subsection{METROLOGY PRE VS POST ENVIRNMENTAL TESTING RESULTS}

As described above in order to determine any observable pre-to-post environmental rigid body change of a given subsystem with associated uncertainty two Monte Carlo transformations were performed using code developed in house (MCTU). These two transformations first brought the pre and post metrology datasets into the same local coordinate system through one subsystem and second determined the rigid body change of the second subsystem. Below are the resultant rigid body changes of all subsystems of interest.

\subsubsection{AOS Motion about the telescope backplane}

The AOS-to-Backplane has a small V1 change though the statistical significance is debatable. There are no observable V2/V3 translations or rotations. 
MCTU 1

RMS of involved target transformation residuals:

\begin{tabular}{|l|l|l|l|l|}
\cline { 2 - 5 } \multicolumn{1}{c|}{} & $\mathbf{u} 1(\mathrm{~mm})$ & $\mathbf{u} 2(\mathrm{~mm})$ & u3 $(\mathrm{mm})$ & Mag $(\mathrm{mm})$ \\
\hline Nominal & 0.01740 & 0.02620 & 0.01840 & 0.03640 \\
\hline MC & 0.01700 & 0.02580 & 0.01860 & 0.03600 \\
\hline
\end{tabular}

MCTU 2

\begin{tabular}{|l|l|l|l|l|}
\cline { 2 - 5 } \multicolumn{1}{c|}{} & $0.1 \mathrm{mrad}=20 \mathrm{arcsec}$ \\
\cline { 2 - 5 } \multicolumn{1}{c|}{} & Nominal $(\mathrm{mm})$ & MC Ave $(\mathrm{mm})$ & U95 $(\mathrm{mm})$ & Nu \\
\hline Translation V1 & -0.0232 & -0.0232 & 0.0077 & 60 \\
\hline Translation V2 & -0.0114 & -0.0114 & 0.0110 & 20 \\
\hline Translation V3 & -0.0124 & -0.0123 & 0.0093 & 27 \\
\hline
\end{tabular}

\begin{tabular}{|l|l|l|l|l|}
\cline { 2 - 5 } \multicolumn{1}{c|}{} & Nominal (Arc Sec) & MC Ave (Arc Sec) & U95 (Arc Sec) & Nu \\
\hline Rotation R1 & -4.9000 & -4.9000 & 3.9000 & 50 \\
\hline Rotation R2 & -1.4000 & -1.4000 & 2.7000 & 18 \\
\hline Rotation R3 & -0.6000 & -0.7000 & 2.7000 & 60 \\
\hline & \multicolumn{5}{|l}{} \\
\cline { 2 - 6 } & Nominal & MC Ave & U95 & Nu \\
\hline Scale & 1.0 & 1.0 & 0.0 & 0 \\
\hline
\end{tabular}

\begin{tabular}{|c|c|c|c|c|}
\hline & \multicolumn{4}{|c|}{ lation residuals: } \\
\hline & u1 $(\mathrm{mm})$ & u2 $(\mathrm{mm})$ & u3 $(\mathrm{mm})$ & Mag(mm) \\
\hline Nominal & 0.00570 & 0.01160 & 0.01410 & 0.01920 \\
\hline MC & 0.00580 & 0.01160 & 0.01400 & 0.01910 \\
\hline
\end{tabular}

\subsubsection{ISIM motion about BSF}

The ISIM-to-BSF MR has a small V1\&V3 change though the statistical significance is debatable. There are no observable V2 translations and no observable rotations.

MCTU 1

\begin{tabular}{|c|c|c|c|c|}
\hline & $\mathrm{u} 1(\mathrm{~mm})$ & u2 $(\mathrm{mm})$ & u3 (mm) & $\operatorname{Mag}(\mathrm{mm})$ \\
\hline Nominal & 0.00900 & 0.02050 & 0.02340 & 0.03230 \\
\hline MC & 0.00900 & 0.02050 & 0.02340 & 0.03230 \\
\hline
\end{tabular}

\begin{tabular}{|c|c|c|c|c|}
\hline & \multicolumn{4}{|c|}{ MCTU 2} \\
\hline & Nominal (mm) & MC Ave (mm) & U95 (mm) & Nu \\
\hline Translation V1 & 0.0378 & 0.0378 & 0.0126 & 60 \\
\hline Translation V2 & 0.0033 & 0.0034 & 0.0141 & 10 \\
\hline \multirow[t]{2}{*}{ Translation V3 } & 0.0460 & 0.0465 & 0.0143 & 59 \\
\hline & Nominal (Arc Sec) & MC Ave (Arc Sec) & U95 (Arc Sec) & $\mathrm{Nu}$ \\
\hline Rotation R1 & -2.8000 & -2.8000 & 2.0000 & 12 \\
\hline Rotation R2 & 5.8000 & 5.8000 & 2.2000 & 60 \\
\hline \multirow[t]{2}{*}{ Rotation R3 } & 3.6000 & 3.6000 & 1.8000 & 27 \\
\hline & Nominal & MC Ave & U95 & $\mathrm{Nu}$ \\
\hline Scale & 1.0 & 1.0 & 0.0 & 0 \\
\hline
\end{tabular}

\begin{tabular}{|c|c|c|c|c|}
\hline & \multicolumn{4}{|c|}{ RMS of involved target transformation residuals: } \\
\hline & $\mathrm{u} 1(\mathrm{~mm})$ & $\mathrm{u} 2(\mathrm{~mm})$ & u3 (mm) & $\operatorname{Mag}(\mathrm{mm})$ \\
\hline Nominal & 0.03260 & 0.02480 & 0.00900 & 0.04190 \\
\hline MC & 0.03220 & 0.02450 & 0.00890 & 0.04150 \\
\hline
\end{tabular}

\subsubsection{SMA Motion about SMM}

There are no observable translations or rotations.

\section{MCTU 1}

\begin{tabular}{|c|c|c|c|c|}
\hline & u1 (mm) & $\mathrm{u} 2(\mathrm{~mm})$ & u3 (mm) & $\operatorname{Mag}(\mathrm{mm})$ \\
\hline Nominal & 0.00420 & 0.00860 & 0.01240 & 0.01570 \\
\hline MC & 0.00380 & 0.00870 & 0.01270 & 0.01590 \\
\hline
\end{tabular}

\begin{tabular}{|c|c|c|c|c|}
\hline & Nominal (mm) & MC Ave (mm) & U95 (mm) & $\mathrm{Nu}$ \\
\hline Translation V1 & -0.0022 & -0.0023 & 0.0163 & 14 \\
\hline Translation V2 & 0.0081 & 0.0083 & 0.0313 & 18 \\
\hline \multirow[t]{2}{*}{ Translation V3 } & 0.0270 & 0.0270 & 0.0246 & 43 \\
\hline & Nominal (Arc Sec) & MC Ave (Arc Sec) & U95 (Arc Sec) & Nu \\
\hline Rotation R1 & 10.9000 & 11.1000 & 9.8000 & 29 \\
\hline Rotation R2 & 4.2000 & 4.2000 & 15.4000 & 36 \\
\hline \multirow{2}{*}{ Rotation R3 } & -10.9000 & -11.3000 & 22.6000 & 12 \\
\hline & Nominal & MC Ave & U95 & $\mathrm{Nu}$ \\
\hline Scale & 1.0 & 1.0 & 0.0 & 0 \\
\hline & \multicolumn{4}{|c|}{ RMS of involved target transformation residuals: } \\
\hline & $\mathrm{u} 1(\mathrm{~mm})$ & $\mathrm{u} 2(\mathrm{~mm})$ & u3 (mm) & $\operatorname{Mag}(\mathrm{mm})$ \\
\hline Nominal & 0.00510 & 0.01090 & 0.02720 & 0.02970 \\
\hline MC & 0.00490 & 0.01060 & 0.02690 & 0.02930 \\
\hline
\end{tabular}




\subsubsection{Extra Credit: ISIM motion about AOS}

The significant optical alignment however is along the light path i.e. how the AOS directs the light into the science instruments house in the ISIM. The metrology plan had previously been written with the thought that a complete USMN of all the significant OTIS targets with reasonable uncertainties would be difficult to achieve. After all the data had been compiled it was observed that the data was, in fact, very good. Below are the results of the science instruments about the tertiary mirror assembly, the ISIM about the AOS. Though there seems to be a measureable change is well below the optically acceptable motion and may be due to the fact that the AOS has a small base and the ISIM is relatively far from the local coordinate system at the base of the AOS thus a large lever arm. If we move the coordinate system to a more favorable place with a larger base, say the ISIM targets this observed translation changes.

\section{MCTU 1}

\begin{tabular}{|c|c|c|c|c|}
\hline & $\mathrm{u} 1(\mathrm{~mm})$ & u2 $(\mathrm{mm})$ & u3 $(\mathrm{mm})$ & $\operatorname{Mag}(\mathrm{mm})$ \\
\hline Nominal & 0.00570 & 0.01160 & 0.01410 & 0.01920 \\
\hline MC & 0.00570 & 0.01160 & 0.01420 & 0.01920 \\
\hline
\end{tabular}

MCTU 2

\begin{tabular}{|l|l|l|l|l|}
\cline { 2 - 5 } \multicolumn{1}{c|}{} & Nominal $(\mathrm{mm})$ & MC Ave $(\mathrm{mm})$ & U95 $(\mathrm{mm})$ & Nu \\
\hline Translation V1 & -0.0274 & -0.0274 & 0.0174 & 22 \\
\hline Translation V2 & 0.0881 & 0.0882 & 0.0313 & 60 \\
\hline Translation V3 & 0.0111 & 0.0113 & 0.0276 & 60 \\
\hline
\end{tabular}

\begin{tabular}{|l|l|l|l|l|}
\cline { 2 - 5 } \multicolumn{1}{c|}{} & Nominal (Arc Sec) & MC Ave (Arc Sec) & U95 (Arc Sec) & Nu \\
\hline Rotation R1 & 2.2000 & 2.2000 & 2.9000 & 60 \\
\hline Rotation R2 & 4.3000 & 4.3000 & 2.7000 & 60 \\
\hline Rotation R3 & 2.2000 & 2.2000 & 2.4000 & 60 \\
\hline & \multicolumn{5}{ll}{} \\
\cline { 2 - 6 } & Nominal & MC Ave & U95 & Nu \\
\hline Scale & 1.0 & 1.0 & 0.0 & 0 \\
\hline
\end{tabular}

\begin{tabular}{|c|c|c|c|c|}
\hline & \multicolumn{4}{|c|}{ RMS of involved target transformation residuals: } \\
\hline & $\mathrm{u} 1$ (mm) & $\mathrm{u} 2(\mathrm{~mm})$ & $\mathrm{u} 3(\mathrm{~mm})$ & $\operatorname{Mag}(\mathrm{mm})$ \\
\hline Nominal & 0.03260 & 0.02480 & 0.00900 & 0.04190 \\
\hline MC & 0.03270 & 0.02500 & 0.00890 & 0.04210 \\
\hline
\end{tabular}

\subsubsection{Delta Gap Metrology}

The average Gap measurements are within 60um average uncertainty with gap uncertainties $>60$ um attributed to the following. Point cloud offset, differences in cloud trimming, point selection, and plane fitting approach among analysts. "Pre" and "Post" crosscheck analysis agreed with primary analysis to within uncertainties. Measured gap absolute delta pre-to-post environmental change results show Ave $=74 \mu \mathrm{m}$ with a $\max =209 \mu \mathrm{m}$. 


\begin{tabular}{|c|c|c|c|c|c|}
\hline \multicolumn{2}{|c|}{ Unit = (mm) } & \multicolumn{4}{|c|}{ "Pre" center section gap'Post" center section gaps } \\
\hline Point1 & Point2 & Average & $\mathbf{U}$ & Average & $\mathbf{U}$ \\
\hline p2_B1 & p2_C1 & 7.030 & 0.041 & 6.824 & 0.052 \\
\hline p3_A1 & p3_B1 & 6.817 & 0.058 & 6.650 & 0.050 \\
\hline p3_A1 & p3_C6 & 7.020 & 0.041 & 7.084 & 0.050 \\
\hline p3_B1 & p3_c6 & 7.010 & 0.046 & 7.061 & 0.065 \\
\hline p4_-A1 & p4늘 1 & 7.083 & 0.041 & 6.988 & 0.051 \\
\hline p4_A1 & $\mathrm{p} 4$ - 1 & 7.083 & $0 . \overline{042}$ & $7 . \overline{183}$ & $0 . \overline{0} 61$ \\
\hline p4_B1 & p4__C1 & 7.670 & 0.043 & 7.654. & 0.056 \\
\hline p5_A6 & $\mathrm{p} 5 \_\mathrm{C} 6$ & 7.004 & $0 . \overline{044}$ & $6 . \overline{804}$ & 0.053 \\
\hline p6_A1 & p6_A6 & 7.028 & 0.053 & 6.972 & 0.057 \\
\hline p6_A1 & p6_c6 & 7.050 & 0.045 & 6.908 & 0.052 \\
\hline p6_A6 & p6_c6 & 7.062 & 0.041 & 6.991 & 0.051 \\
\hline p7_A1 & p7_A2 & 7.173 & 0.043 & 7.278 & 0.051 \\
\hline p7_A1 & p7_C1 & 6.992 & 0.044 & 7.045 & 0.051 \\
\hline p7_A2 & p7_C1 & 7.125 & 0.042 & 7.024 & 0.058 \\
\hline p8_A2 & p8_c1 & 7.109 & 0.061 & 7.098. & 0.051 \\
\hline p9 & p9_A6 & 7.180 & 0.057 & 6.971 & 0.052 \\
\hline p10_A1 & p10_A2 & $7 . \overline{151}$ & $0 . \overline{047}$ & $7 . \overline{130}$ & 0.050 \\
\hline p11_A5 & p11_A6 & 6.978 & 0.042 & 6.978 & 0.051 \\
\hline p12_A5 & p12_A6 & 7.075 & 0.083 & 6.921 & 0.050 \\
\hline p13_A2 & p13_A3 & 6.938 & 0.043 & 6.952 & 0.050 \\
\hline p14_A2 & p14_A3 & 7.028 & 0.052 & 7.092 & 0.053 \\
\hline p15_A4 & $\underline{p} 15$ A & 7.129 & 0.042 & 7.026 & 0.054 \\
\hline p16 & p16 14 & 7.109 & 0.052 & 7.003 & 0.054 \\
\hline p17_A5 & p17_c4 & 7.128 & 0.040 & 7.144 & 0.051 \\
\hline p18_A4 & p18_A5 & 7.228 & 0.053 & 7.069 & 0.052 \\
\hline p18_A4 & p18_C4 & 7.058 & 0.087 & 6.923 & 0.056 \\
\hline p18_A5 & p18_C4 & 7.077 & 0.044 & 7.043 & 0.065 \\
\hline
\end{tabular}

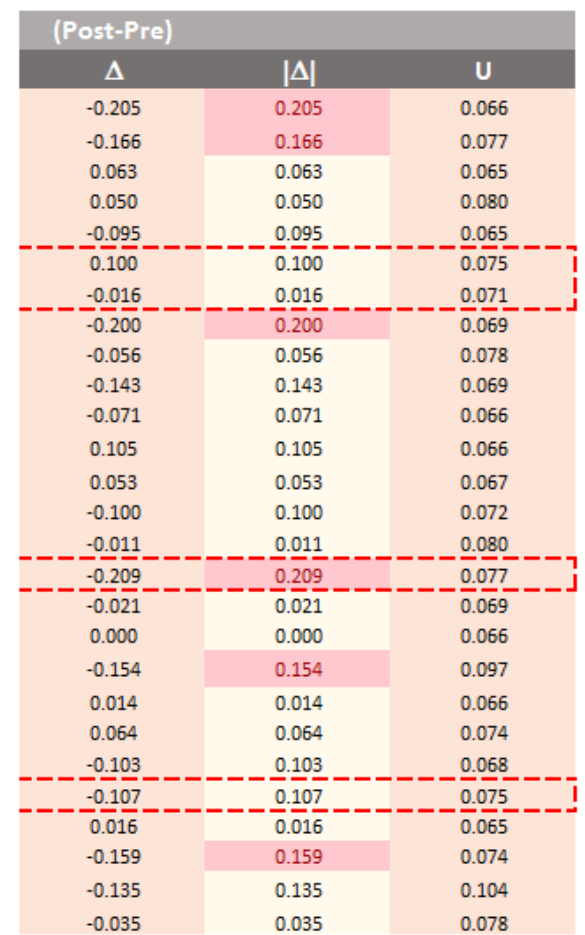

\begin{tabular}{|c|c|c|c|c|c|c|c|c|c|}
\hline \multicolumn{2}{|c|}{ Unit = (mm) } & \multicolumn{4}{|c|}{ "Pre" center section gap'spost" center section gaps } & \multicolumn{4}{|c|}{ (Post-Pre) } \\
\hline Point1 & Point2 & Average & $\mathbf{U}$ & Average & $\mathbf{U}$ & & $\Delta$ & $|\Delta|$ & $\mathbf{U}$ \\
\hline p19_A3 & p19_A4 & 7.026 & 0.054 & 6.898 & 0.057 & & -0.128 & 0.128 & 0.079 \\
\hline p19_A3 & p19_C3 & 7.079 & 0.040 & 7.114 & 0.057 & & 0.035 & 0.035 & 0.069 \\
\hline p19_A4 & p19_C3 & 7.135 & 0.042 & 7.139 & 0.054 & & 0.004 & 0.004 & 0.069 \\
\hline p20_A3 & p20_C3 & 6.955 & 0.043 & 7.061 & 0.052 & & 0.107 & 0.107 & 0.068 \\
\hline p21_A4 & p21_B4 & 6.830 & 0.042 & 6.801 & 0.051 & & -0.029 & 0.029 & 0.066 \\
\hline p21_A4 & p21_C4 & 7.153 & 0.041 & 7.081 & 0.052 & & -0.072 & 0.072 & 0.067 \\
\hline p21_B4 & p21_C4 & 6.956 & 0.044 & 6.924 & 0.052 & & -0.032 & 0.032 & 0.068 \\
\hline p22_A4 & p22_B4 & 6.967 & 0.040 & 6.950 & 0.054 & & -0.017 & 0.017 & 0.068 \\
\hline p22_A4 & p22_C3 & 7.124 & 0.043 & 7.141 & 0.053 & & 0.017 & 0.017 & 0.068 \\
\hline$\underline{p} 22=$ & p22__C 3 & 7.068 & 0.043 & 7.007 & 0.059 & & -0.061 & 0.061 & $\underline{0.073}$ \\
\hline p23 B4 & p23_c4 & 6.985 & 0.064 & 6.881 & 0.052 & & -0.104 & 0.104 & 0.082 \\
\hline \multirow[t]{2}{*}{ p24_B4 } & p24_C3 & 7.201 & 0.047 & 7.158 & 0.050 & & -0.044 & 0.044 & 0.069 \\
\hline & \multicolumn{5}{|c|}{ "Pre" center section gap'spost" center section gaps } & & (Post-Pr & & \\
\hline Point1 & Point2 & Average & $\mathbf{U}$ & Average & $\mathbf{U}$ & & $\Delta$ & $|\Delta|$ & $\mathbf{U}$ \\
\hline wp1_B2 & wp1_C2 & 7.497 & 0.042 & 7.450 & 0.051 & & -0.047 & 0.047 & 0.066 \\
\hline wp2_B2 & wp2_C2 & 7.019 & 0.046 & 7.009 & 0.053 & & -0.010 & 0.010 & 0.070 \\
\hline wp3 $3=$ B3 & wp3 3 C2 2 & 7.113 & 0.043 & 7.015 & 0.052 & & -0.097 & 0.097 & 0.068 \\
\hline \multirow[t]{2}{*}{ wp4 $=$} & wp4_C2 & 7.039 & 0.040 & 6.917 & 0.057 & & -0.122 & 0.122 & 0.070 \\
\hline & \multicolumn{5}{|c|}{ "Pre" center section gapspost" center section gaps } & & (Post-Pr & & \\
\hline Point1 & Point2 & Average & $\mathbf{U}$ & Average & $\mathbf{U}$ & & $\Delta$ & $|\Delta|$ & $\mathbf{U}$ \\
\hline wp5_B6 & wp5_c5 & 6.997 & 0.042 & 7.052 & 0.051 & & 0.054 & 0.054 & 0.067 \\
\hline wp6_B6 & wp6_c5 & 7.012 & 0.041 & 7.032 & 0.050 & & 0.020 & 0.020 & 0.065 \\
\hline wp7_B5 & wp7_c5 & 7.114 & 0.040 & 7.126 & 0.054 & & 0.012 & 0.012 & 0.067 \\
\hline \multirow[t]{4}{*}{ wp8_B5 } & wp8_c5 & 7.067 & 0.040 & 7.040 & 0.057 & & -0.027 & 0.027 & 0.069 \\
\hline & & Ave & 0.047 & Ave & 0.054 & Ave & -0.044 & 0.074 & 0.072 \\
\hline & & Max & 0.087 & Max & 0.065 & Max & 0.107 & 0.209 & 0.104 \\
\hline & & $\min$ & 0.040 & $\min$ & 0.050 & $\min$ & -0.209 & 0.000 & 0.065 \\
\hline
\end{tabular}

\subsection{SUMMARY}

We successfully performed pre and post environmental metrology on the OTIS subsystems in local coordinates. The results of this metrology showed minimal to no relative rigid body motions between subsystems as well as no individual 
PMSA gap deltas as a result of vibration and acoustic testing. To within uncertainty of the measurements the OTIS passed the testing of survivability of launch for both physical and optical performance.

\section{REFERENCES}

[1] Joseph F. Sullivan et.al, JWST's optical telescope simulator for verification of the Integrated Science Instrument Module Proc. SPIE. 9951, Optical System Alignment, Tolerancing, and Verification X, 99510E. (September 27, 2016) doi: $10.1117 / 12.2237834$

[2] Scott R. Antonille et.al, Optical testing and verification methods for the James Webb Space Telescope Integrated Science Instrument Module element, Proc. SPIE. 9951, Optical System Alignment, Tolerancing, and Verification X, 995105. (September 27, 2016) doi: 10.1117/12.2238838

[3] Theo Hadjimichael et.al, Alignment of the James Webb Space Telescope Integrated Science Instrument Module Element Proc. SPIE. 9951, Optical System Alignment, Tolerancing, and Verification X, 99510C. (October 18, 2016) doi: $10.1117 / 12.2238829$

[4] David L. Aronstein et.al, Wavefront-error performance characterization for the James Webb Space Telescope (JWST) Integrated Science Instrument Module (ISIM) science instruments, Proc. SPIE. 9904, Space Telescopes and Instrumentation 2016: Optical, Infrared, and Millimeter Wave, 990409. (July 29, 2016) doi: 10.1117/12.2233842

[5] Scott O. Rohrbach et.al, Stray light modeling of the James Webb Space Telescope (JWST) Integrated Science Instrument Module (ISIM) , Proc. SPIE. 9947, Current Developments in Lens Design and Optical Engineering XVII, 99470K. (September 28, 2016) doi: 10.1117/12.2238827

[6] Dave Kubalak et.al, JWST science instrument pupil alignment measurements, Proc. SPIE. 9951, Optical System Alignment, Tolerancing, and Verification X, 99510D. (September 27, 2016) doi: 10.1117/12.2238830

[7] A. Slotwinski and P. Blanckaert, "Frequency Modulated Coherent Laser Radar Technology," Proceedings of the OPTIMESS2007 Workshop, Leuven, Belgium, May 28-30, 2007.

[8] J. Burge, P. Su, C. Zhao, and T. Zobrist, "Use of a commercial laser tracker for optical alignment," Optical System Alignment and Tolerancing, J. Sasian and M. Ruda, eds., Proc. SPIE 6676, SPIE Press, Bellingham, 66760E-1-12, 2007.

[9] New River Kinematics, Inc., Williamsburg, Va.

[10] S. Sandwith and R. Predmore, "Real-time 5-Micron Uncertainty with Laser Tracking Interferometer Systems using Weighted Trilateration," Proc. SPIE.

[11] Spatial Analyzer Users Manual, New River Kinematics, Williamsburg, v. 1.21.2008,

[12] Joseph Hayden et.al, Monte Carlo Method for Uncertainty Propagation in JWST Metrology Databases, CMSC May 212015 15:41. 